\title{
Inhibition of Hyperpolarization-Activated Cation Current in Medium-Sized DRG Neurons Contributed to the Antiallodynic Effect of Methylcobalamin in the Rat of a Chronic Compression of the DRG
}

\author{
Ming Zhang, ${ }^{1}$ Wenjuan Han, ${ }^{1}$ Jianyong Zheng, ${ }^{2}$ Fancheng Meng, \\ Xiying Jiao, ${ }^{1}$ Sanjue $\mathrm{Hu},{ }^{1}$ and Hui $\mathrm{Xu}^{1}$ \\ ${ }^{1}$ Institute of Neurosciences, the Fourth Military Medical University, Xian 710032, China \\ ${ }^{2}$ State Key Laboratory of Cancer Biology and Institute of Digestive Diseases, Department of Digestive Surgery, \\ Xijing Hospital, the Fourth Military Medical University, Xian 710032, China \\ Correspondence should be addressed to Hui Xu; xubz@fmmu.edu.cn
}

Received 23 January 2015; Revised 23 March 2015; Accepted 23 March 2015

Academic Editor: Gianluca Coppola

Copyright ( $\odot 2015$ Ming Zhang et al. This is an open access article distributed under the Creative Commons Attribution License, which permits unrestricted use, distribution, and reproduction in any medium, provided the original work is properly cited.

Recently several lines of evidence demonstrated that methylcobalamin (MeCbl) might have potential analgesic effect in experimental and clinical studies. However, it was reported that $\mathrm{MeCbl}$ had no effect on treating lumbar spinal stenosis induced pain. Thus, the effects of short-term and long-term administration of $\mathrm{MeCbl}$ were examined in the chronic compression of dorsal root ganglion (CCD) model. We found that mechanical allodynia was significantly inhibited by a continuous application of high dose and a single treatment of a super high dose of $\mathrm{MeCbl}$. Little is known about mechanisms underlying the analgesia of $\mathrm{MeCbl}$. We examined the effect of $\mathrm{MeCbl}$ on the spontaneous activity (SA), the excitability, and hyperpolarization-activated nonselective cation ion current in compressed medium-sized dorsal root ganglion (DRG) neurons using extracellular single fiber recording in vivo and whole-cell patch clamp in vitro. We found that MeCbl significantly inhibited the SA of A-type sensory neurons in a dose-dependent manner and inhibited the excitability of medium-sized DRG neurons. In addition, MeCbl also decreased $I_{h}$ current density in injured medium-sized DRG neurons. Our results proved that MeCbl might exert an analgesic effect through the inhibition $I_{h}$ current and then might inhibit the hyperexcitability of primary sensory neurons under neuropathic pain state.

\section{Introduction}

Methylcobalamin $(\mathrm{MeCbl})$ has a strong affinity for nerve tissues $[1,2]$. Furthermore, MeCbl participates in DNA and protein methylation as a coenzyme of methionine synthase in the transmethylating action [3-5]. MeCbl plays an important role in myelination, neuronal differentiation and replication, and cellular activity in nerve tissues [6, 7]. Combined with other medicines, MeCbl has always been used to treat B12 deficiency and Alzheimer's disease syndromes [8,9]. Evidence showed that the coapplication of $\mathrm{MeCbl}$ and pioglitazone instinctively decreased allodynia and hyperalgesia in diabetic rats [10], and the combination of $\mathrm{MeCbl}$ and vitamin $\mathrm{E}$ alleviated thermal hyperalgesia in sciatic nerve crush injured rats [11]. Very few studies reported that MeCbl treatment relieved paresthesia, burning pain, and spontaneous pain in neuropathic patients $[12,13]$ and $\mathrm{MeCbl}$ alleviated subacute herpetic, trigeminal, and glossopharyngeal neuralgia [14-16]. Several clinical trials confirmed the efficacy and safety of $\mathrm{MeCbl}$ in relieving pain and improving axonal degeneration and nerve conduction velocities for diabetic peripheral neuropathy $[17,18]$. But there was evidence that $\mathrm{MeCbl}$ lacked effectiveness in treating lumbar spinal stenosis induced pain [19]. In addition, the precise mechanism of MeCbl's effect on peripheral neuropathy remains obscure. In the present study, we evaluated the effect of monotherapy of $\mathrm{MeCbl}$ in the chronic compression of dorsal root ganglion (CCD) model, which mimics low back pain and lumbar spinal stenosis syndromes well. 
Mounting evidence suggests that a possible cause of low back pain and radicular pain is the mechanical deformation of the dorsal root ganglion (DRG) and its nerve roots, resulting from spinal stenosis, radiculopathies, and tumors [20]. The animal model of CCD mimics low back pain and radicular pain syndromes in the rat [21]. Previous studies showed that DRG neurons exhibited hyperexcitability, including spontaneous activity, increased excitability, decreased potassium current, and upregulation of the hyperpolarizationactivated cation current $\left(I_{h}\right)$ in the CCD rat [22-25].

The hyperexcitability of DRG neurons associated with an injury of a peripheral nerve or the sensory ganglion may contribute to pain-related behaviors in experimental animal models of neuropathic pain. $I_{h}$ regulates neuronal excitability by limiting membrane hyperpolarization and facilitating depolarization $[24,26] . I_{h}$ exists widely in primary sensory neurons $[27,28] . I_{h}$ blocker ZD7288 inhibited the SA originated from injured DRG neurons in animal models of SNL and CCI rats [29-31]. Many medium-sized sensory neurons are responsive to thermal and/or mechanical noxious stimuli through peripheral nociceptors. Others transmit nonnociceptive information as low-threshold mechanoreceptors [32]. The low-threshold type of medium-sized DRG neurons might not be normally involved in acute pain but might contribute to tactile allodynia in pathological cases. Thus, the effect of MeCbl on the SA, the excitability, and $I_{h}$ in mediumsized DRG neurons were further determined in CCD rats in the present study.

\section{Materials and Methods}

2.1. Animals and Surgery. Adult female Sprague-Dawley (SD, $120 \mathrm{~g}-250 \mathrm{~g}, n=132$ ) rats were used in experiments, according to the guildlines for the care and use of laboratory animals and the protection committee of our university. The CCD model was prepared according to the previous method [21]. The sterilized surgical procedures were carried out under sodium pentobarbital anesthesia $(40 \mathrm{mg} / \mathrm{kg}$, i.p.). The skin was incised on the left side of the lumbar vertebrae between L4 and L6 and the left paraspinal muscles were separated from the mammillary process and the transverse process at the L4-L6 level. In the first group of rats (the CCD group), the L5 intervertebral foramen was clearly exposed and a fine, Lshaped needle inserted about $4 \mathrm{~mm}$ into the L5 intervertebral foramen at a $30^{\circ}$ angle with respect to the dorsal middle line and $10^{\circ}$ with respect to the vertebral horizontal line. When the needle tip reached the DRG, the hind leg muscles of the operated side exhibited a slight, transient twitch. Then, the needle was withdrawn from the L5 intervertebral foramen and a stainless steel rod, $4 \mathrm{~mm}$ in length and with different diameters, was inserted into the L5 intervertebral foramen along the path of the needle. The diameter of the stainless steel rod was increased in relation to the weight of the rat; for example, a diameter of $0.4 \mathrm{~mm}$ was selected for the rats of $120-150 \mathrm{~g}$ and a diameter of $0.5 \mathrm{~mm}$ and $0.6 \mathrm{~mm}$ for the rats of 151-200 $\mathrm{g}$ and 201-250 $\mathrm{g}$, respectively [21]. Then the muscular layer and skin were sutured. To isolate recording and administrating pools for the experiments of single-fiber recording, L5 DRG was compressed in the rat. To obtain more intact DRG samples for the whole-cell patch clamp recording, both L4 and L5 DRGs were compressed in the rat. As for the sham group, the surgical procedure was identical to that for the CCD group, except that the L-shaped needle was not inserted into the intervertebral foramen.

2.2. Pain Behavioral Test of Mechanical Allodynia. Fifty-two SD rats were housed in a plastic cage $(40 \mathrm{~cm} \times 60 \mathrm{~cm} \times 30 \mathrm{~cm})$ with soft bedding and free access to food and water and $12 \mathrm{~h}$ day/12 h night cycle. These animals were divided randomly into CCD and sham groups.

Rats were placed in plastic cage with metal mesh floor and allowed to habituate for at least $30 \mathrm{~min}$ before testing. Mechanical stimuli, produced by Von Frey filaments (Stoelting Co., USA), were used to assess the bilateral paw withdrawal threshold. Every rat was placed on a metal mesh floor in a plastic cage $(20 \mathrm{~cm} \times 25 \mathrm{~cm} \times 15 \mathrm{~cm})$. A series of von Frey filaments (1.0-15.0 g) touched the plantar part of the hindpaw in the ascending order [33]. Flicking, withdrawal, or licking of the injured hindpaw was treated as positive response to nociception. Each filament was applied 5 times to its bending force, and a paw withdrawal threshold was defined as the minimal force of filaments inducing three or more positive responses. In order to prevent the tissue from injury, the cut-off threshold was assigned at $15.0 \mathrm{~g}$. Dosage of $\mathrm{MeCbl}$ injection solution (Eisai, Japan) was administrated according to rat's weight. To study the short-term effect of $\mathrm{MeCbl}$ on neuropathic pain, $\mathrm{MeCbl}$ was intraperitoneally injected to CCD rats on the 3rd postoperative day for one time. $\mathrm{MeCbl}(1.25,2.5$, and $10 \mathrm{mg} / \mathrm{kg})$ and vehicle $(2.5$ and $10 \mathrm{mg} / \mathrm{kg}$ ) were, respectively, injected in CCD and sham rats. $\mathrm{MeCbl}$ was also intraperitoneally and successively injected every day from the 3rd postoperative day.

Mannitol (Chemscene, Japan) is the supplementary material in the $\mathrm{MeCbl}$ injection. The maximal concentration of Mannitol was considered as vehicle in the experiment.

\subsection{Electrophysiological Recording}

2.3.1. Extracellular Recording of A-Type Single-Fiber Activities of L5 Dorsal Root In Vivo. The spontaneous activity of DRG single fibers was obtained from CCD rats. All animals at postoperative 3-7 days were used for extracellular recording of single fiber in vivo. Rats were anesthetized with mixture of anesthetics ( $\alpha$-chloralose $20 \mathrm{mg} / \mathrm{kg}$ and urethane $340 \mathrm{mg} / \mathrm{kg}$, i.p.). The laminectomy was performed at L1-L2 and L4-L5 and a small pool was prepared at each of the two sites. In the L4-L5 pool, the stainless steel rod was taken out and the compressed L5 DRG was sufficiently exposed. The spinal nerve was transected about $5-10 \mathrm{~mm}$ distal to the ipsilateral L5 DRG so that the discharges were recorded from L5 dorsal root fibers originated primarily from the L5 ganglion and not from receptors in peripheral tissue. During the experiment, the L4-L5 pool was filled with $1 \mathrm{~mL}$ of warm Kreb's solution $\left(35-37^{\circ} \mathrm{C}\right.$ ) containing (in $\mathrm{mM}$ ) $\mathrm{NaCl} 150, \mathrm{KCl}$ 5, $\mathrm{CaCl} 2$ 2, $\mathrm{MgCl} 21, \mathrm{D}$-glucose 10, and HEPES 10, with the $\mathrm{pH}$ adjusted to 7.4. In the L1-L2 pool, the dorsal root of the L5 DRG was covered with warm paraffin oil $\left(35-37^{\circ} \mathrm{C}\right)$. Point thermometer (TM-1320, OBEST) was applied to detect 
temperature of two pools during experiment. And the rats lay on the linear animal temperature regulator (BME-461A, CAMS) which was kept at $37^{\circ} \mathrm{C}$. Under the microscope, a bundle of L5 dorsal root (about $10 \mu \mathrm{m}$ in diameter) which might include one microfilament or a few nerve fibers was isolated and cut off. Then the broken end of fractured bundle was located to a fine platinum electrode $(29 \mu \mathrm{m}$ in diameter) for electrophysiological recording. The firing patterns of Atype fiber bundle were displayed in a memory oscilloscope (VC-11, Japan) and collected via an A/D board to a computer hard drive and stored for offline analysis. Unitary firings were identified according to their spike waveforms, and those microfilaments with single-fiber firings were used for further recording $[21,34]$.

The spontaneous activity of A-type dorsal root was recorded for no less than $3 \mathrm{~min}$, and then $\mathrm{MeCbl}$ or vehicle was administrated to L5 DRG, respectively. The mean firing rate of about $3 \mathrm{~min}$ before application of $\mathrm{MeCbl}$ was used as the basal frequency of SA. The inhibitory rate of $\mathrm{MeCbl}$ or vehicle was calculated by the formula: inhibitory rate $=$ (basal frequency of SA in the absence of $\mathrm{MeCbl}$ or vehicle - the frequency of $\mathrm{SA}$ in the presence of $\mathrm{MeCbl}$ or vehicle)/basal frequency of SA $\times 100 \%$. The percentage changes of firing rates were considered significant if the changes were $15 \%$ or greater [34].

2.3.2. Whole-Cell Patch Clamp Recording of Medium-Sized DRG Neurons In Vitro. CCD rats of postoperative 3-7 days and sham rats were anesthetized with pentobarbital sodium ( $40 \mathrm{mg} / \mathrm{kg}$, i.p.). L4 and L5 DRGs on the left were carefully exposed and the spinal nerve connected to DRG was reserved as long as possible $(>2 \mathrm{~cm})$ in the preparation. Intact L 4 and L5 DRGs and attached spinal nerve were carefully removed from the vertebral column and placed into artificial cerebrospinal fluid (ACSF (in mM): $\mathrm{NaCl} 124, \mathrm{KCl} 2.5, \mathrm{NaH}_{2} \mathrm{PO}_{4}$ 1.2, $\mathrm{MgCl}_{2}$ 1.0, $\mathrm{CaCl}_{2} 2, \mathrm{NaHCO}_{3} 25$, and Glucose 10). Spinal dura maters and connective tissues nearby were cleaned, and then the intact ganglia were digested in a digestive solution ( $2 \mathrm{mg} / \mathrm{mL}$ trypsin and $3.2 \mathrm{mg} / \mathrm{mL}$ type-A collagenase) for $45 \mathrm{~min}$ at $37^{\circ} \mathrm{C}$ and were agitated by gentle bubbling with mixed gas $\left(95 \% \mathrm{O}_{2}\right.$ and $\left.5 \% \mathrm{CO}_{2}\right)$. The intact ganglia were transferred into ACSF and incubated in ACSF with mixed gas at $28^{\circ} \mathrm{C}$ for at least 1 hour before being moved into the recording chamber.

During the recording, the chamber was filled with different solutions. These bath solutions (ACSF) were saturated with $95 \% \mathrm{O}_{2}$ and $5 \% \mathrm{CO}_{2}$. The end of the spinal nerve was sucked by a stimulating electrode. And the conduction velocity $(\mathrm{CV})$ of medium-sized DRG soma was measured as the distance between the stimulating electrode and the recording electrode divided by the time between the electrical stimulus and the evoked AP. Individual neurons could be visualized with a $40 \times$ water-immersion objective under a microscope (BX51WI; Olympus, Tokyo, Japan). Whole-cell current and voltage clamp recording were carried out by a multiclamp 700A amplifier (Molecular Devices Corporation, Sunnyvale, CA, USA). Patch pipettes (2-4M $\Omega$ ) were pulled from borosilicate glass capillaries and pulled on a micropipette puller (P-97, Sutter Instrument, USA). Neurons, in which membrane potentials were more negative than $-50 \mathrm{mV}$ and which exhibited overshooting action potentials, were selected for further study. Signals were digitized at $10 \mathrm{kHz}$ by a $1320 \mathrm{~A} / \mathrm{D}$ board (Molecular Devices, USA) and used for data acquisition and analysis. A gigaohm seal was reached by a small negative pressure, and the whole-cell recording was established by further negative pressure or a buzz signal. The membrane potential was held at $-60 \mathrm{mV}$ in the voltage clamp. And the data were discarded if the $\mathrm{Ra}$ or membrane potential changed by $20 \%$ during an experiment.

The DRG soma was classified visually by the diameter of its soma as small $(<30 \mu \mathrm{m})$, medium-sized $(31-45 \mu \mathrm{m})$, or large $(>45 \mu \mathrm{m})$ [22]. Medium-sized DRG somas were selected in our experiment. Passive and active membrane features were collected. Resting membrane potential, Cm (membrane capacitance), and Rm (membrane resistance) were recorded on line. Positive membrane features including AP amplitude, AP half-width, max-rise slope, and AHP amplitude were analyzed on the first evoked AP. The first action potential was evoked by the step wave from $-100 \mathrm{pA}$ to $1000 \mathrm{pA}$ with an increase of $50 \mathrm{pA}$. AP amplitude $(\mathrm{mV})$ was regarded as voltage difference between the resting membrane potential (RMP) and the peak upward. AP half-width was characterized as duration of AP after 50\% repolarization. Max-rise slope was measured as differential on AP; the value of the peak point and AHP amplitude $(\mathrm{mV})$ was voltage difference between the lowest point of hyperpolarization and the RMP.

In our experiment, the internal solution contained (in $\mathrm{mM}) \mathrm{K}$-gluconate $120, \mathrm{KCl} 18, \mathrm{CaCl}_{2} 1, \mathrm{MgCl}_{2}$ 2, EGTA 5, HEPES $10, \mathrm{Na}_{2}$-ATP, and $\mathrm{Na}_{3}$-GTP. Osmolarity was adjusted to $280-290 \mathrm{mOsm}(\mathrm{pH}=7.4)$. The hyperpolarization potentials were got from $-110 \mathrm{mV}$ to $-60 \mathrm{mV}$ with an increment of $10 \mathrm{mV}$. In order to evaluate changes of $I_{h}$ between CCD and sham rats, $I_{h}$ current density was calculated as $I_{h}$ current amplitude divided by $\mathrm{Cm}$. MeCbl was perfused to intact DRGs for $5 \mathrm{~min}$, and then the indices above were measured.

2.4. Statistics. All data were stored in computer hard drives for offline analysis and were expressed as mean \pm sem. The P-clamp 9 software (Molecular Devices, USA) was used for whole-cell patch clamp data acquisition and analysis. Statistical evaluations were performed by using Statistical Product and Service Solutions (SPSS) software. The data were analyzed and painted by the software of sigmaplot (10.0), origin (8.0), and Coreldraw (X3). The change of SA, $I_{h}$ current density, and passive and active membrane properties of medium-sized DRG neurons before and after the treatment of $\mathrm{MeCbl}$ were analyzed by Student's $t$-test (paired sample $t$-test). The number of APs, $I_{h}$ current density, and passive and active membrane properties were compared by one-way analysis of variance (one-way ANOVA) between CCD and sham groups. One-way ANOVA was also used to test significant difference on inhibition rate at different concentration of MeCbl. Repeated-measures ANOVA with Bonferroni's post hoc test were used to compare the number of APs and mechanical behavior before and after the application of $\mathrm{MeCbl}$. The pairwise comparison in the number of APs and mechanical behavior between CCD and sham groups were 
analyzed using multivariate analysis of variance (MANOVA). $P<0.05$ was assumed to indicate statistical significance.

\section{Results}

3.1. Effects of MeCbl on Mechanical Allodynia. The mechanical paw withdrawal thresholds (PWTs) of CCD $(n=6)$ and sham rats $(n=6)$ were recorded for 21 days. After the surgery, both ipsilateral and contralateral PWTs of CCD rats $(n=6)$ were much decreased compared to those of sham rats during postoperative 21 days $(P s<0.001$, repeatedmeasures ANOVA). MeCbl was intraperitoneally injected during postoperative 3 to 10 days, and short-term and longterm applications of $\mathrm{MeCbl}$ on mechanical allodynia were investigated.

3.1.1. A Short-Term Treatment of MeCbl Alleviated Mechanical Allodynia. Following the single intraperitoneal injection of $\mathrm{MeCbl}$, pain behavioral tests were examined in $1 \mathrm{~h}, 3 \mathrm{~h}$, $5 \mathrm{~h}, 7 \mathrm{~h}$, and $24 \mathrm{~h}$. There were no significant differences in ipsilateral and contralateral PWTs of CCD rats after the shortterm application of $\mathrm{MeCbl}(1.25$ and $2.5 \mathrm{mg} / \mathrm{kg})$ and vehicle $(2.5 \mathrm{mg} / \mathrm{kg})(P s>0.05$, repeated-measures ANOVA, $n=$ 5, Figure 1(a)). Moreover, MeCbl $(2.5 \mathrm{mg} / \mathrm{kg})$ did not affect bilateral PWTs of sham group compared to those of vehicle $(P s>0.05$, repeated-measures ANOVA, Figure 1(b)). The single treatment of a higher dose of $\mathrm{MeCbl}(10 \mathrm{mg} / \mathrm{kg})$ was tested in $20 \mathrm{~min}$ to $30 \mathrm{~min}$ of the injection. Both ipsilateral and contralateral PWTs were enhanced in CCD rats following the injection of $\mathrm{MeCbl}(P s<0.05$, paired $t$-test, Figure $1(\mathrm{c}))$. But vehicle did not significantly affect bilateral PWTs $(P s>$ 0.05 , paired $t$-test, Figure $1(\mathrm{c}))$.

3.1.2. A Continuous Treatment of MeCbl Ameliorated Mechanical Allodynia. MeCbl (1.25 mg/kg) was intraperitoneally injected continuously and once daily for 28 days, and there were no significant differences in bilateral PWTs between CCD and sham groups $(P s>0.05$, repeated-measures ANOVA, $n=5$, Figure 2(a)), while $\mathrm{MeCbl}(2.5 \mathrm{mg} / \mathrm{kg})$ significantly increased bilateral PWTs compared to vehicle $(P s<$ 0.001 , repeated-measures ANOVA, $n=5$, Figure 2(a)). Since the successive injection of $\mathrm{MeCbl}(2.5 \mathrm{mg} / \mathrm{kg})$ for 5 days, bilateral PWTs were distinctly increased, compared to those of the vehicle group $(P s<0.01$, repeated-measures ANOVA, $n=5$, Figure 2(a)).

Effects of $\mathrm{MeCbl}(2.5 \mathrm{mg} / \mathrm{kg})$ and vehicle (Mannitol $2.5 \mathrm{mg} / \mathrm{kg}$ ) were also studied on sham rats. MeCbl and Mannitol did not significantly affect bilateral PWTs of sham rats compared to vehicle group $(P s>0.05$, repeated-measures ANOVA, Figure 2(b)).

\subsection{Inhibitory Effects of $\mathrm{MeCbl}$ on the SA of A-Type DRG Neurons}

3.2.1. MeCbl Inhibited the SA of A-Type DRG Neurons In Vivo. Among 62 units of microfilament of dorsal root exhibited stabled SA in CCD rats ( $n=42$, CCD rats), $66.1 \%(41 / 62)$ of those units were sensitive to $\mathrm{MeCbl}$. The insensitive effect was considered as the percentage changes of firing rates lower than $15 \%$ in the presence of the maximal dose of $\mathrm{MeCbl}$ $(300 \mu \mathrm{M})$. Eighty-two point nine percent (34/41) could be recovered in $10 \mathrm{~min}$ after the washout (Figure 3(a)). MeCbl inhibited the SA in a dose-dependent manner $(n=5)$ from 3 to $300 \mu \mathrm{M}$ in CCD rats $(P s<0.05$, Student's $t$-test, Figure $3(\mathrm{~b}))$. The $50 \%$ inhibition concentration of $\mathrm{MeCbl}$ was $76 \mu \mathrm{M}$ (Figure 3(c)).

\subsubsection{MeCbl Inhibited the SA of Medium-Sized DRG Neurons} In Vitro. For A-type DRG neurons were recorded in the in vitro experiment; the medium-sized DRG neurons were selected from CCD and sham rats (CCD group: $37.7 \pm$ $0.62 \mu \mathrm{m}, n=36$; sham group: $36.05 \pm 0.57 \mu \mathrm{m}, n=23)$. The conduction velocities of medium-sized DRG neurons were measured $(11.20 \pm 2.94 \mathrm{~m} / \mathrm{s}, n=7)$, which belonged to A $\delta$ DRG neurons. Among the $7 \mathrm{~A} \delta$ neurons, $4 \mathrm{~A} \delta$ neurons belong to CCD group and $3 \mathrm{~A} \delta$ neurons come from sham group. Twenty-two point two (8/36) medium-sized neurons from the intact DRGs developed SA in vitro. After the application of $\mathrm{MeCbl}(10 \mu \mathrm{M})$, the SA was eliminated during $60 \mathrm{~s}$ and the SA was recovered in $10 \mathrm{~min}$ after washout. About $50 \%$ of MeCbl-responsive neurons were reversible in medium-sized DRG neurons of CCD rats after washout (Figure 3(d)).

\subsection{Inhibitory Effects of MeCbl on the Excitability of Medium-Sized DRG Neurons}

3.3.1. MeCbl Inhibited the Excitability of Medium-Sized DRG Neurons. The excitability of medium-sized DRG neurons was recorded in intact DRGs in vitro $(P>0.05$, one-way ANOVA, CCD group: $38.91 \pm 1.09 \mu \mathrm{m}, n=17$; sham group: $35.51 \pm 0.54 \mu \mathrm{m}, n=15)$. No significant differences were found in passive membrane properties between the sham and CCD groups ( $P s>0.05, n=32$, Student's $t$-test, Table 1). And $\mathrm{MeCbl}$ did not affect passive membrane properties in sham rats either ( $P s>0.05, n=15$, Student's $t$-test, Table 1$)$.

Compared to sham rats, AP amplitude and max-rise slope were both markedly increased ( $P s<0.05, n=32$; one-way ANOVA, Figures 4(a) and 4(b)); AP half-width and AHP amplitude were obviously decreased $(P s<0.05$, one-way ANOVA, $n=32$, Figures $4(\mathrm{c})$ and $4(\mathrm{~d}))$. The increases of AP amplitude and AP max-rise slope and the decreases of AP half-width and AHP amplitude of medium-sized DRG neurons were reversed in the presence of $\mathrm{MeCbl}$ in CCD rats $(P s<0.001$, Student's $t$-test, $n=17$, Figures $4(\mathrm{a})-$ $4(\mathrm{~d}))$. Nevertheless, MeCbl did not influence active membrane properties including AP amplitude, max-rise slope, and AP half-width and AHP amplitude of medium-sized DRG neurons in sham rats $(P s>0.05, n=15$, Student's $t$-test, Figure 4).

The number of APs was significantly increased in the CCD group than that of sham group $(P s<0.05$, one-way ANOVA, Figures 5(a) and 5(b)). The increased number of APs was remarkably decreased by the administration of MeCbl in CCD group $(P s<0.05$, repeated-measures ANOVA, Figures 5(a) and 5(b)), but not in the sham group 

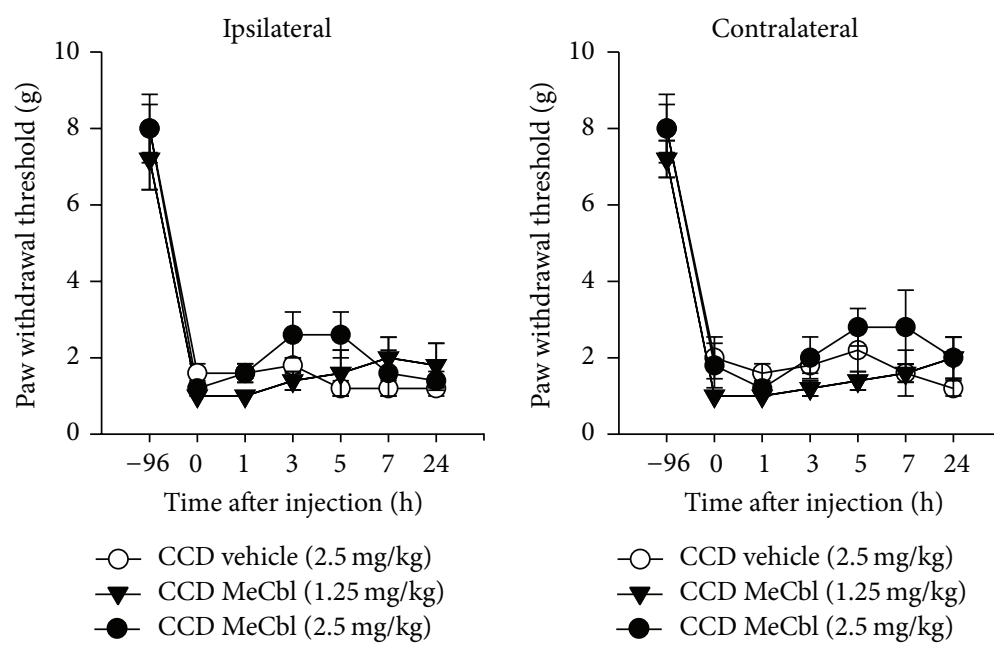

(a)
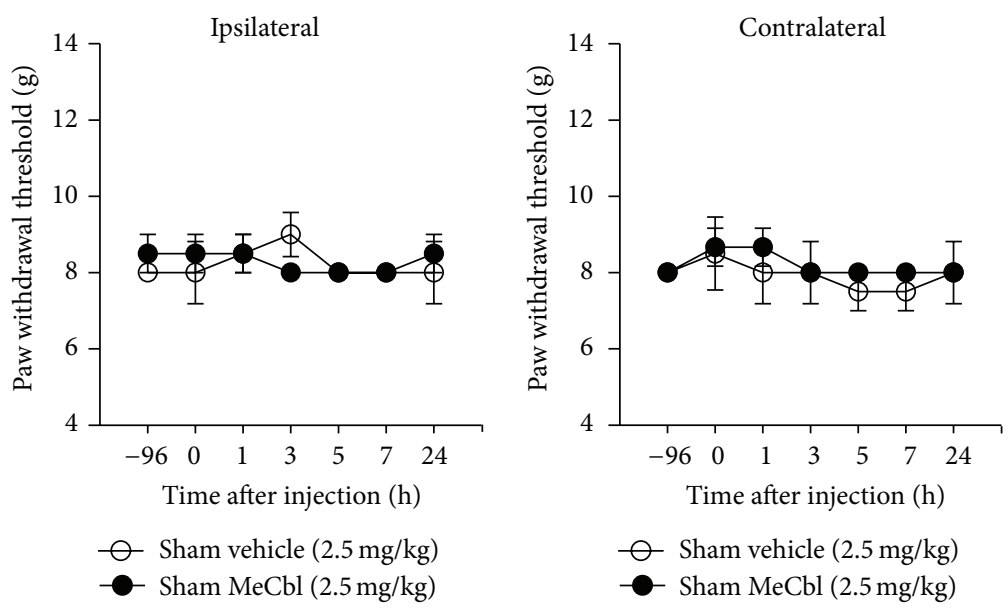

(b)
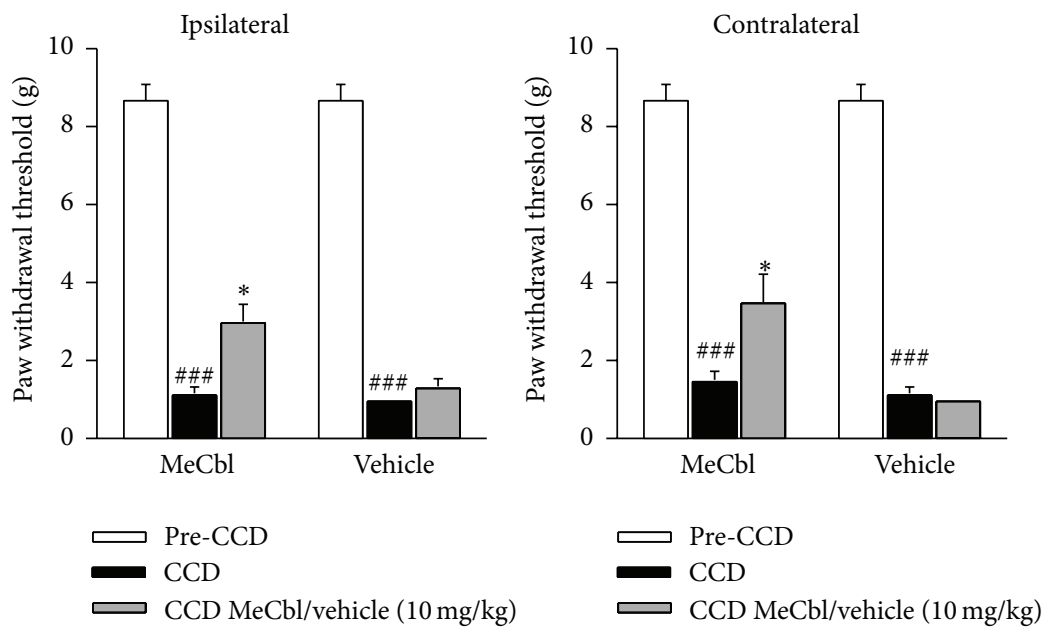

(c)

FIGURE 1: High dose of MeCbl ameliorated mechanical allodynia of rats following the CCD in the short term. MeCbl (1.25 mg/kg, $2.5 \mathrm{mg} / \mathrm{kg}$, and $10 \mathrm{mg} / \mathrm{kg}$ ) was injected intraperitoneally on the postoperative 3 to 10 days. (a) Effects of MeCbl on ipsilateral and contralateral mechanical paw withdrawal thresholds in CCD rats following the injection. (b) No effects of MeCbl on ipsilateral and contralateral mechanical paw withdrawal thresholds in sham rats. (c) A higher concentration of MeCbl affected bilateral paw withdrawal thresholds. ${ }^{\# \#} P<0.001$, compared to those in pre-CCD group; ${ }^{*} \mathrm{P}<0.05$, compared with those of CCD group. 
Ipsilateral

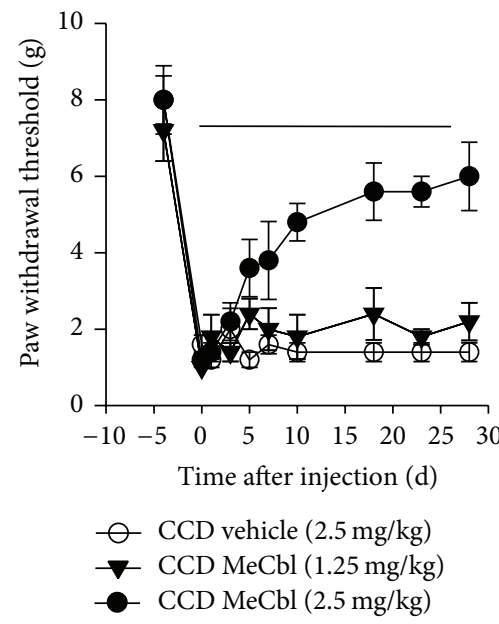

Contralateral

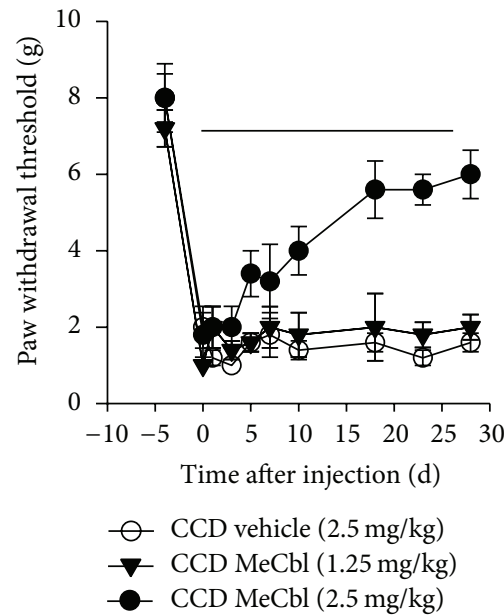

(a)

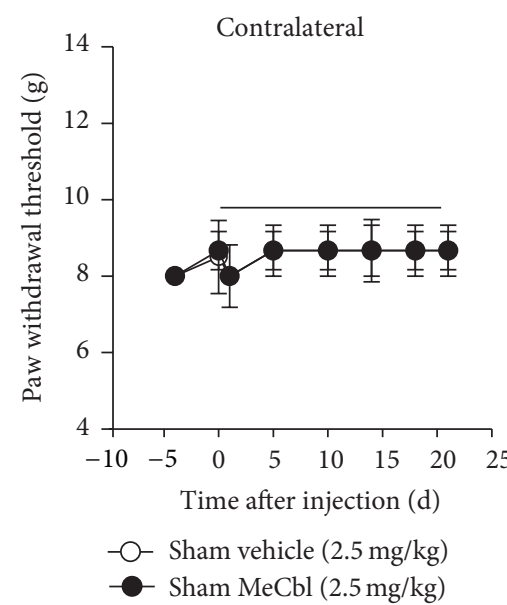

(b)

Figure 2: Continuous treatment of MeCbl inhibited bilateral mechanical allodynia of CCD rats. Continuous treatment of MeCbl (1.25 mg/kg and $2.5 \mathrm{mg} / \mathrm{kg}$ ) or Mannitol $(2.5 \mathrm{mg} / \mathrm{kg}$ ) was injected intraperitoneally from the 3rd postoperative day (black line segment). (a) Effects of $\mathrm{MeCbl}$ on bilateral mechanical paw withdrawal thresholds of CCD rats. (b) Effects of MeCbl on mechanical paw withdrawal thresholds of sham rats.

TABLE 1: The effect of MeCbl $(10 \mu \mathrm{M})$ on passive membrane properties of medium-sized DRG neurons.

\begin{tabular}{lcccc}
\hline & $n$ & $\begin{array}{c}\text { Membrane } \\
\text { potential }(\mathrm{mV})\end{array}$ & $\mathrm{Cm}(\mathrm{pF})$ & $\mathrm{Rm}(\mathrm{M} \Omega)$ \\
\hline Sham & 15 & $-69.00 \pm 1.36$ & $64.14 \pm 10.93$ & $49.37 \pm 8.19$ \\
Sham + & 15 & $-66.20 \pm 1.77$ & $65.83 \pm 10.68$ & $48.24 \pm 8.17$ \\
MeCbl & & & & \\
CCD & 17 & $-66.45 \pm 1.22$ & $86.00 \pm 19.16$ & $62.74 \pm 10.91$ \\
CCD + & 17 & $-64.12 \pm 1.86$ & $77.01 \pm 8.84$ & $52.32 \pm 8.08$ \\
MeCbl & & & \\
\hline
\end{tabular}

No significant differences in membrane potential, $\mathrm{Cm}$ (membrane capacitance), and $\mathrm{Rm}$ (membrane resistance) in medium-sized DRG neurons of $\mathrm{CCD}$ rats in the presence of $\mathrm{MeCbl}$ (Ps $>0.05$, Student's $t$-test).

$(P s>0.05$, repeated-measures ANOVA, Figures 5(a) and $5(\mathrm{~b}))$.
3.3.2. $\mathrm{MeCbl}$ Inhibited the Increase of $I_{h}$ Current Density in Medium-Sized DRG Neurons. About 65\% DRG A-type neurons of CCD and sham groups expressed $I_{h}$ current. $I_{h}$ current was abolished by ZD7288 $(10 \mu \mathrm{M})$ but could not be reversed. And it could be absolutely abolished by $2 \mathrm{mM} \mathrm{Cs}^{2+}$ ( $n=4$, data not shown) in $5 \mathrm{~min}$ and was recovered in $20 \mathrm{~min}$. In contrast to sham group, $I_{h}$ current densities of mediumsized DRG neurons were increased markedly in CCD rats $(P<0.05$, one-way ANOVA, Figures 6(a) and 6(b)).

$I_{h}$ current of medium-sized DRG neurons was recorded after the application of $\mathrm{MeCbl}$ in vitro (Figure 6(b)). $I_{h}$ current density was significantly decreased by the perfusion of MeCbl in CCD rats $(n=7, P<0.05$, Student's $t$-test, Figure 6(c)). However, $I_{h}$ current density was not affected by the perfusion of $\mathrm{MeCbl}$ in sham rats $(n=7, P<0.05$, Student's $t$-test, Figure 6(c)). 

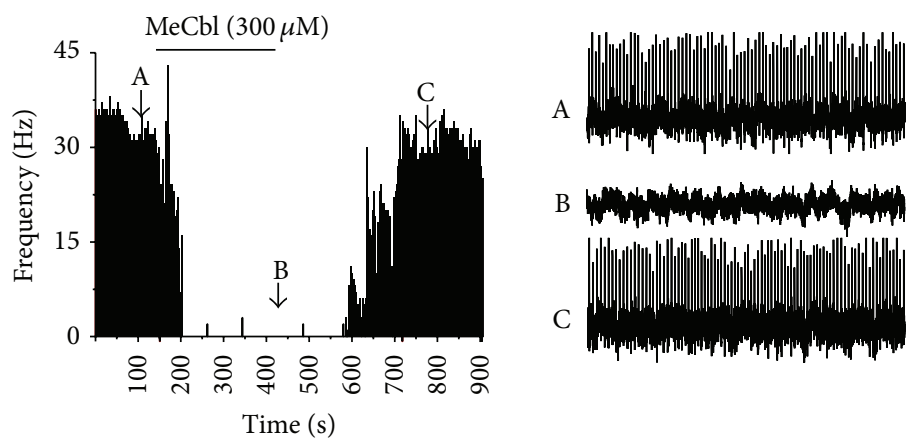

(a)

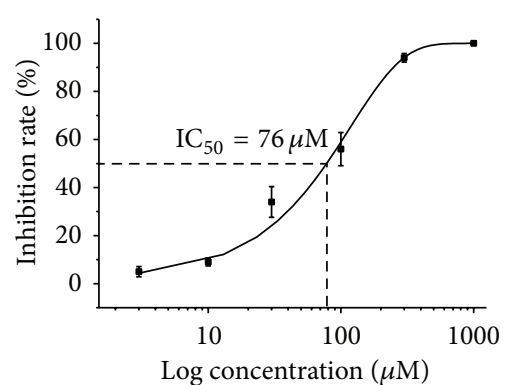

(c)

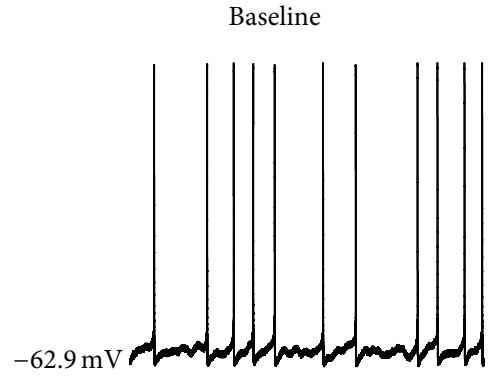

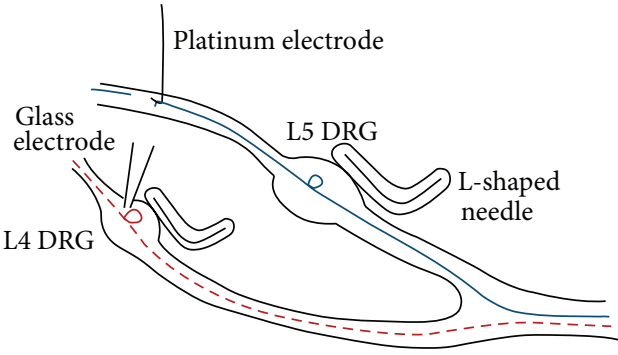

(d)
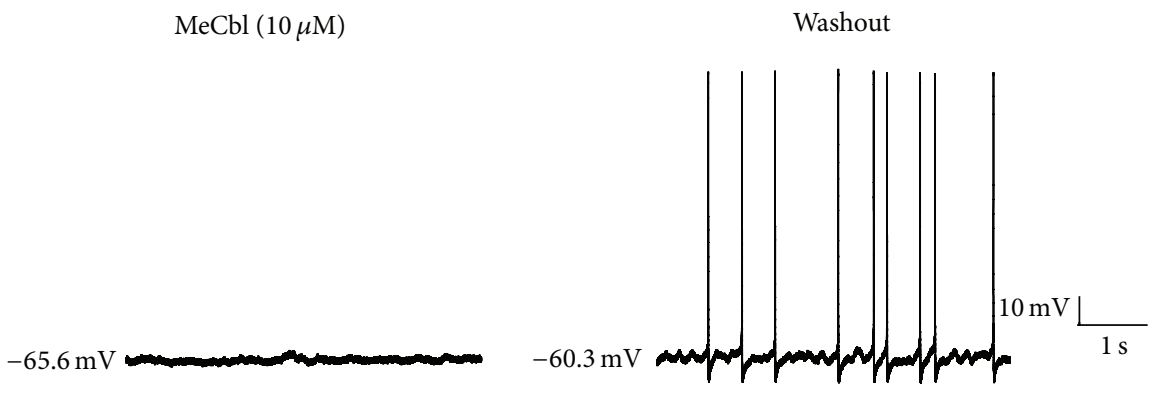

(e)
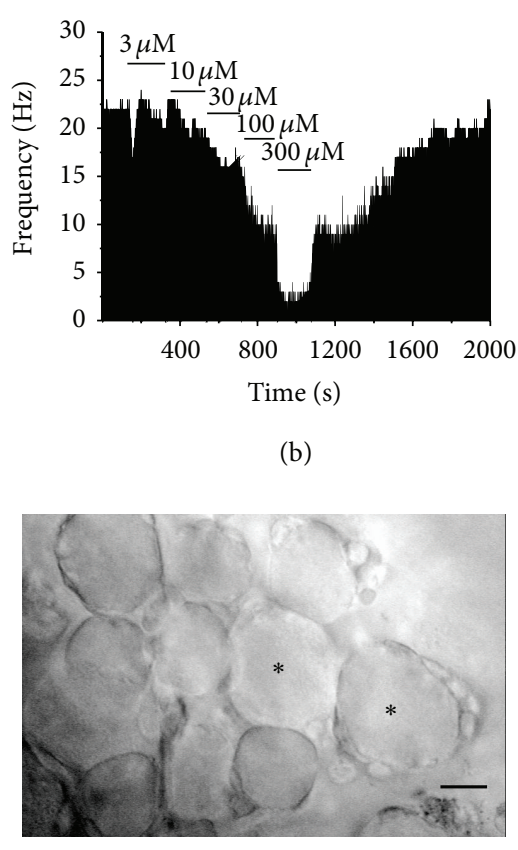

(b)

FIGURE 3: MeCbl inhibited spontaneous activity (SA) of A-type neurons of the compressed DRG in a dose-dependent way. (a) Time histogram shows that the application of $\mathrm{MeCbl}(300 \mu \mathrm{M})$ decreases the basal firing rate of an A-type dorsal root fiber in vivo. Three traces in right panel were showed firing patterns before (A), during (B) and wash (C) the application of MeCbl. (b) Time histogram shows the inhibitory effect of $\mathrm{MeCbl}$ in a dose-dependent manner. (c) The concentration-response curve shows the value for $\mathrm{IC}_{50}(76 \mu \mathrm{M})$ of MeCbl. (d) Schematic graph of whole-cell recording of DRG neurons and photography of medium-sized DRG neurons. Scale $=20 \mu \mathrm{m}$. (e) MeCbl (10 $\mu \mathrm{M})$ inhibited the SA of one medium-sized DRG neuron of CCD group.

\section{Discussion}

In the present study, we demonstrated that MeCbl inhibited mechanical allodynia in the rat CCD model after a long-term administration and a higher concentration of a short-term treatment of MeCbl. Moreover, MeCbl inhibited the SA, the excitability, and $I_{h}$ of medium-sized DRG neurons following the CCD. It is likely that $\mathrm{MeCbl}$ may exhibit analgesic effect through inhibiting $I_{h}$ and hyperexcitability of injured DRG neurons.

4.1. Continuous Treatment of MeCbl Ameliorated Mechanical Allodynia. MeCbl participates in nervous system maintenance through transmethylating action. Previous studies displayed that $\mathrm{MeCbl}$ has widely been used in the treatment of nervous system disorders including diabetic peripheral neuropathy due to promoting myelination and nerve regeneration [35]. Continuous treatment with ultrahigh dose of $\mathrm{MeCbl}$ promoted nerve regeneration and myelination [36, 37]. In the present study, we used CCD model, which mimics low back pain and lumbar spinal stenosis syndromes well, to evaluate the effect of monotherapy of MeCbl. Continuous administration of a dose of $\mathrm{MeCbl}(2.5 \mathrm{mg} / \mathrm{kg} / \mathrm{day})$ higher than that used in previous reports $(500 \mu \mathrm{g}-1 \mathrm{mg} / \mathrm{kg} /$ day) was used. Our results showed that a continuous treatment of high dose MeCbl markedly alleviated tactile allodynia in the CCD rat, while low and medium dose MeCbl could not. Moreover, a short-term administration of high dose $\mathrm{MeCbl}$ 


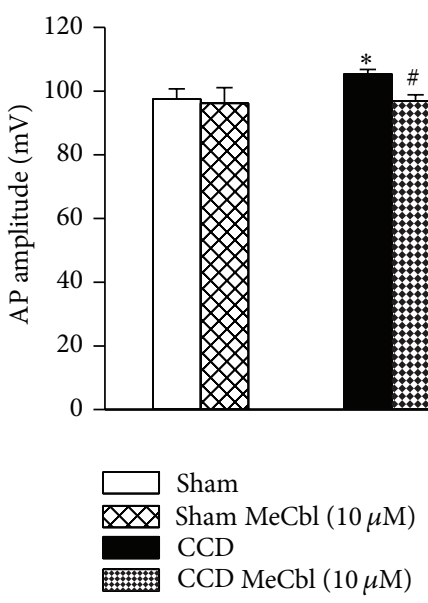

(a)

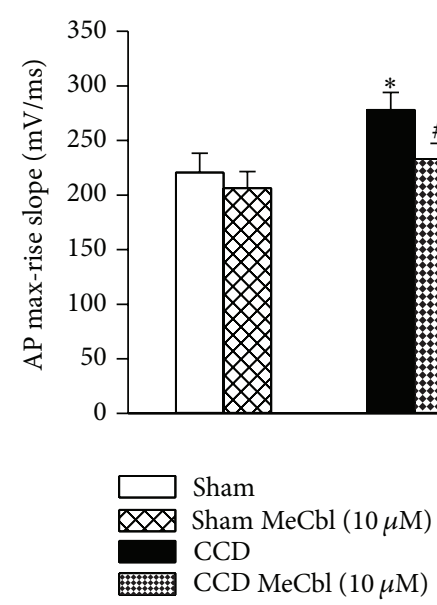

(b)

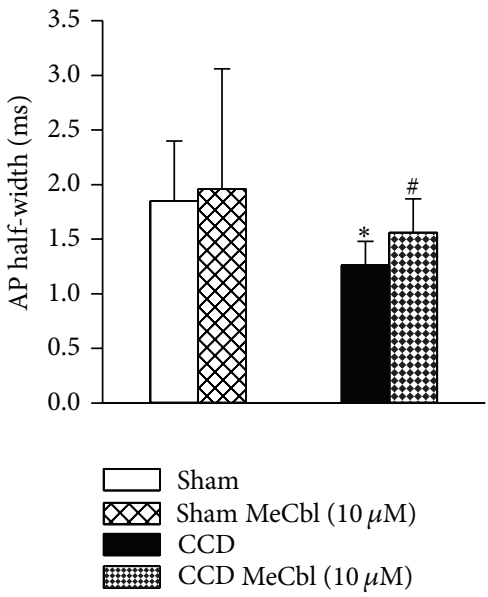

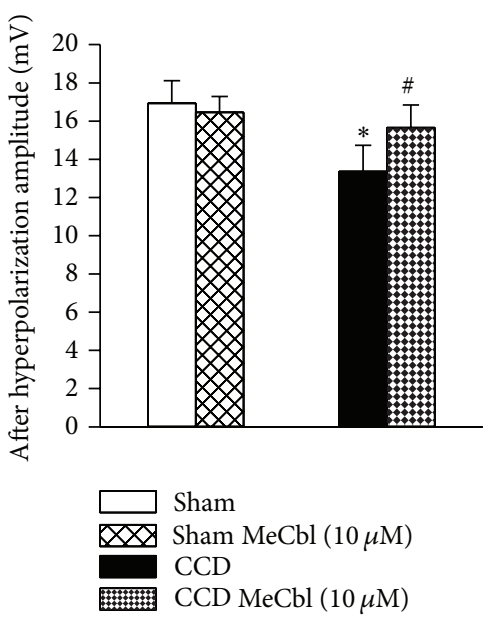

(c)

(d)

FIGURE 4: Effects of MeCbl on active membrane properties of medium-sized DRG neurons. Effects of MeCbl on active membrane properties of medium-sized DRG neurons. (a) AP amplitude; (b) AP max-rise slope; (c) AHP amplitude; (d) AP half-width. ${ }^{*} P<0.05$, significant differences in active membrane properties of medium-sized DRG neurons compared to those in the absence of MeCbl group; ${ }^{*} P<0.05$, compared with those of sham group.

also exhibited an analgesic effect on mechanical allodynia. Thus, our results here confirmed similar results that continuous administration of high dose MeCbl could ameliorate neuropathic pain associated with diabetic neuropathy $[12$, 13] and also showed that the single treatment of an extra high dose of $\mathrm{MeCbl}$ ameliorated mechanical allodynia. But our results were not consistent with previous report that vitamin $\mathrm{B} 12$ or the coapplication of $\mathrm{MeCbl}$ and vitamin $\mathrm{E}$ did not alter mechanical allodynia in sciatic nerve crush injured rats $[11,38]$. The discrepancy is very likely due to different doses and periods of $\mathrm{MeCbl}$ treatment as only high dose and long-term treatment of $\mathrm{MeCbl}$ may be effective in treating mechanical allodynic behavior. It is proposed that mechanical hyperalgesia may involve different mechanisms from those of the thermal hyperalgesia. Further studies are necessary to address the different efficacy of $\mathrm{MeCbl}$ on thermal hyperalgesia and mechanical allodynia.
4.2. MeCbl Inhibited the SA and the Excitability of A-Type DRG Neurons. MeCbl acts as a coenzyme of methionine synthase, which transforms homocysteine to methionine in the methylation cycle including methylation of DNA or proteins [5]. Although several studies showed that $\mathrm{MeCbl}$ improved nerve regeneration and nerve conduction velocity via altering activation of cellular signaling pathway in peripheral neuropathy models, precise analgesic mechanism of $\mathrm{MeCbl}$ is still needed to be clarified. For example, continuous administration of high doses of $\mathrm{MeCbl}$ not only facilitated neurite outgrowth and neuronal survival but also improves nerve regeneration and functional recovery through activation of protein kinases extracellular regulated protein kinases 1/2 (Erk1/2) and protein kinase B (Akt) in sciatic nerve injury model [6]. MeCbl upregulated the expression of neural insulin-like growth factor-1 (IGF-1) gene [39]. MeCbl normalized altered PKC activities in experimental diabetic neuropathy[40]. 

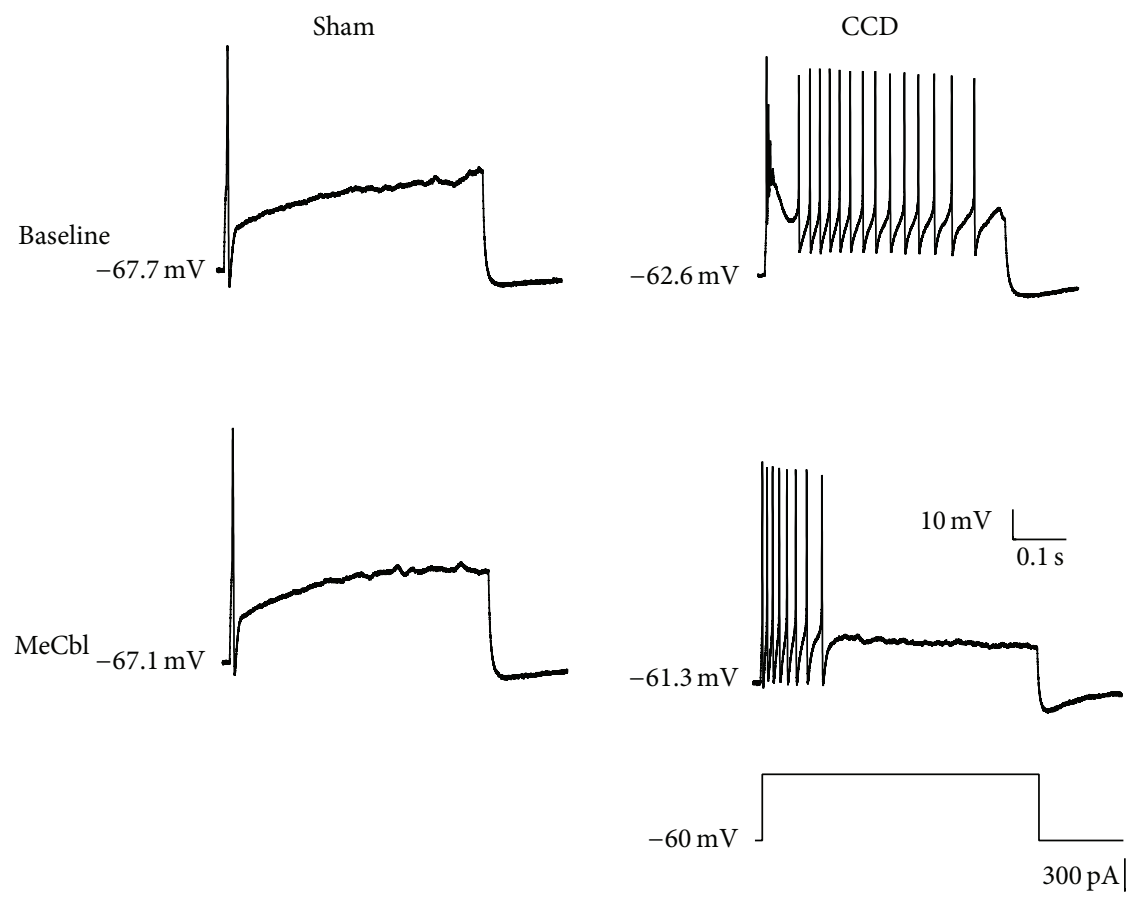

(a)
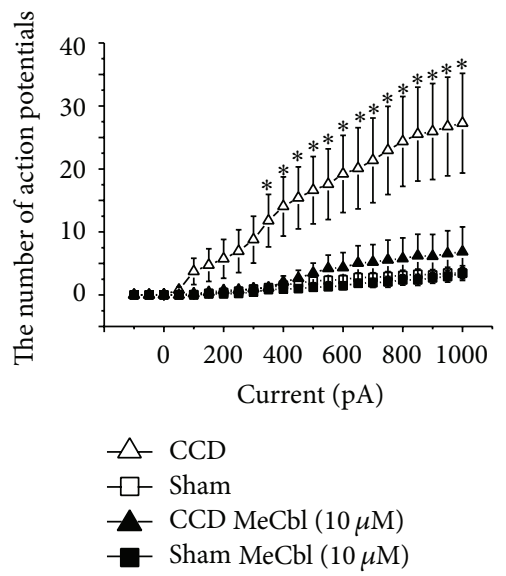

(b)

FIgURE 5: MeCbl inhibited the number of APs of medium-sized DRG neurons. (a) Original traces of APs induced by the depolarized square wave in absence and the presence of $\mathrm{MeCbl}$. (b) Inhibitory effect of $\mathrm{MeCbl}$ on the number of action potentials of medium-sized DRG neurons. ${ }^{*} P<0.05$, compared to those in the absence of MeCbl group.

However, the downstream target of signaling pathway of $\mathrm{MeCbl}$ remains obscure.

Previous studies showed that DRG neurons exhibited the hyperexcitability in the CCD rat, such as SA, increased excitability, and upregulated $I_{h}$ [22-25]. Our study showed that continuous treatment of MeCbl exerted an antiallodynic behavior in the CCD rat. The low-threshold type of mediumsized DRG neurons may become sensitized after tissue inflammation or peripheral nerve injury [41-43]. Thus, the effect of MeCbl was further determined on the SA and the excitability of medium-sized DRG neurons. Our results provided the experimental evidence that $\mathrm{MeCbl}$ significantly inhibited the SA of A-type dorsal root fibers in a dosedependent way in vivo and the SA from medium-sized DRG neurons in vitro from the CCD rat. It indicated that the analgesic mechanism of $\mathrm{MeCbl}$ improved mechanical allodynia through inhibiting the SA of medium-sized DRG neurons and A-type dorsal root fibers in neuropathic pain state.

Recently, several studies showed that medium-sized DRG neurons exhibited hyperexcitability, such as an increased sodium current, a decreased delayed rectifier voltagedependent potassium current, and a decreased threshold for action potential $[25,44]$. According to conduction velocity, medium-sized DRG neurons recorded were considered as A $\delta$ DRG neurons in the present study [45]. Our results showed that the amplitude and the max-rise slope of AP were significantly increased, and AP half-width and AHP amplitude were markedly decreased in medium-sized DRG neurons of CCD rats. It is consistent with LaMotte's reports that excitability of compressed medium-sized DRG neurons might be obviously increased $[25,44]$. Increased max-rise slope of AP and AP amplitude and decreased AP half-width may be due to an increased sodium current in medium-sized DRG neurons following the compression of the DRG. It is reported that AHP amplitude was augmented by $I_{h}$ inhibitor ZD7288 [46]. Our results provide new evidence that AHP amplitude was decreased in medium-sized DRG neurons, indicating an increased $I_{h}$ in those neurons of CCD rats.

Our results showed that MeCbl significantly decreased these increased characters of AP amplitude and the max-rise slope in CCD rats, and increased the decrease of AP halfwidth and AHP amplitude as well. And MeCbl obviously suppressed the number of APs induced after nerve injury. It was suggested that $\mathrm{MeCbl}$ inhibited the increased excitability of medium-sized DRG neurons in CCD rats. The half-life of vitamin B12 in serum ranges from approximately 20 to $50 \mathrm{~min}$, and one administration may not maintain a high concentration of vitamin B12 [47]. Therefore, to maintain the serum concentration of vitamin B12 to some extent, we continuously administered a higher dose. Our results showed an analgesic effect on mechanical allodynia following a single injection of an extra high dose $\mathrm{MeCbl}$, while continuous treatment of minimal MeCbl may overcome the fast degeneration and maintain a high serum concentration of MeCbl. Therefore, continuous treatment of a lower dose of $\mathrm{MeCbl}$ showed an obvious and a stable analgesic efficiency. Combined with behavioral data, our results suggested that continuous treatment of high dose $\mathrm{MeCbl} \mathrm{might}$ 

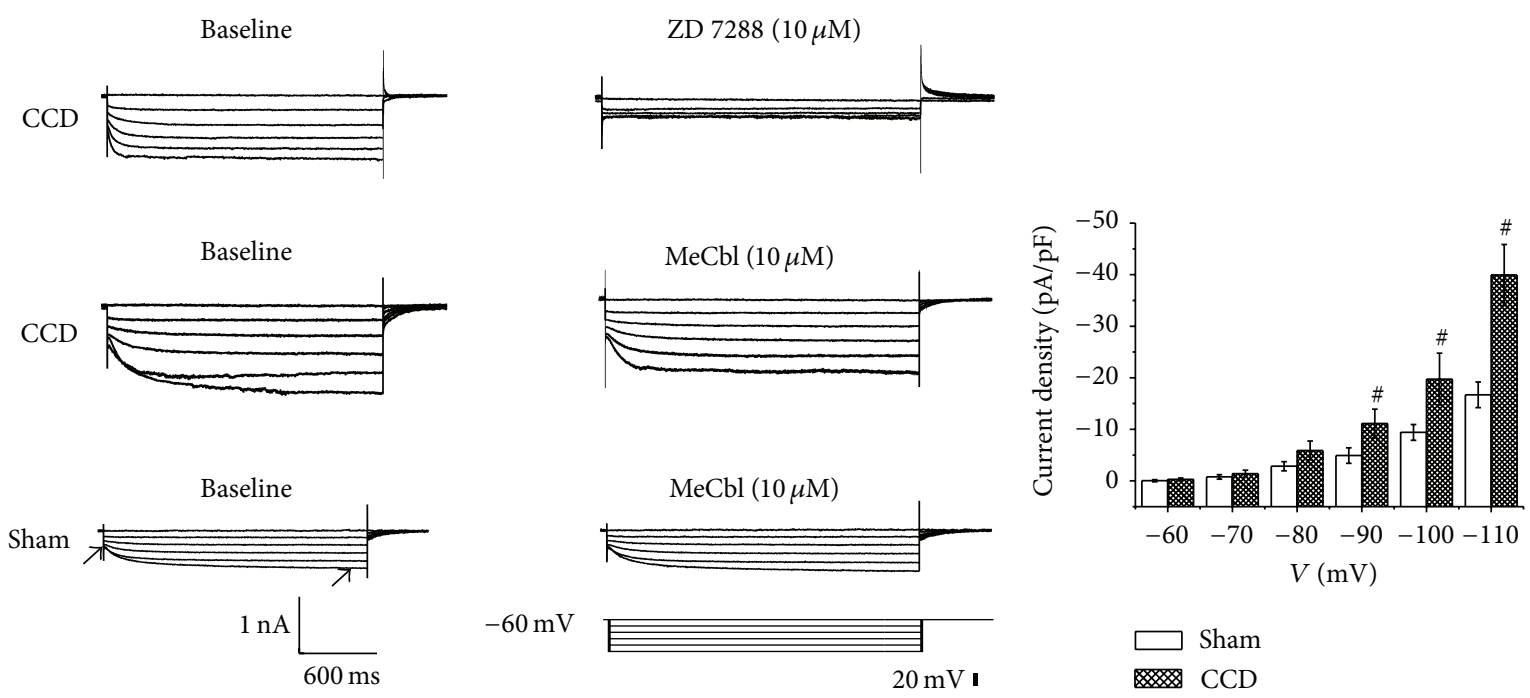

(a)

(b)

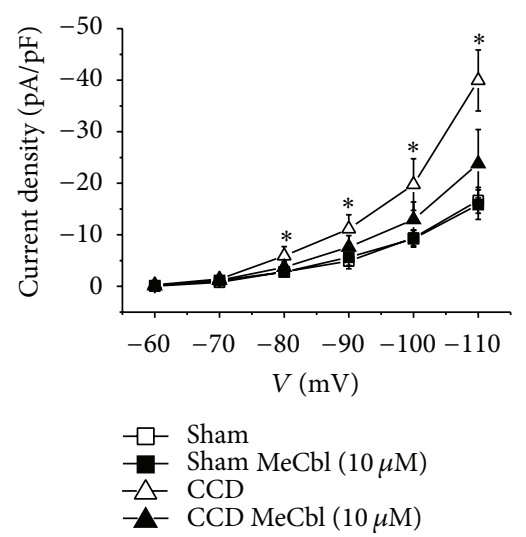

(c)

FIGURE 6: MeCbl inhibited the $I_{h}$ of medium-sized neurons of DRG neurons. (a) $I_{h}$ current is evoked by hyperpolarizing voltage steps from a holding potential of $-60 \mathrm{mV}$. $I_{h}$ current amplitude is calculated from the difference between the steady-state and the instantaneous current. (b) Increased $I_{h}$ current density of medium-sized DRG neurons in CCD rats. ${ }^{\#} P<0.05$, compared with those of sham group. (c) Effects of $\mathrm{MeCbl}$ on the $I_{h}$ current density of medium-sized DRG neurons. ${ }^{*} \mathrm{P}<0.05$, compared with those in the absence of MeCbl.

exhibit an antiallodynia via inhibiting the hyperexcitability of medium-sized DRG neurons in neuropathic pain state.

\subsection{MeCbl Inhibited $I_{h}$ in Medium-Sized DRG Neurons.} $I_{h}$ has been implicated in nociception and chronic pain. The ion channels underlying $I_{h}$ are the hyperpolarizationactivated cyclic nucleotide-gated (HCN) family [48]. HCN channels are activated by membrane hyperpolarization, are permeable to sodium and potassium ions, and comprise four isoforms, HCN1-HCN4. HCN1 and HCN2 contribute to different amplitude and properties of $I_{h}$ in nociceptive or low-threshold mechanoreceptive sensory neurons $[49,50]$. Previous studies showed that $I_{h}$ was upregulated in mediumsized DRG neurons [24, 29]. More recent studies showed increased HCN2 subunit mediated mechanical allodynia after peripheral nerve injury and inflammation [51, 52]. Our results showed that the magnitude of $I_{h}$ was increased significantly in medium-sized DRG of CCD rats, consistent with other studies. Furthermore our results showed that $\mathrm{MeCbl}$ significantly reversed the decrease of AHP amplitude and the increase of $I_{h}$ in medium-sized DRG neurons. It is suggested that MeCbl may inhibit $I_{h}$ and then suppress the SA and the hyperexcitability of medium-sized DRG neurons in neuropathic pain state. MeCbl may exhibit antiallodynia through inhibiting $I_{h}$ and may further inhibit the SA and the hyperexcitability of injured medium-sized DRG neurons. $\mathrm{MeCbl}$ participates in nervous system maintenance through the methylation of DNA or proteins. It is very likely that MeCbl inhibited the $I_{h}$, the hyperexcitability of mediumsized DRG neurons in an epigenetic modulation. Therefore, the mechanism underlying the inhibition of $\mathrm{MeCbl}$ on $I_{h}$ and the hyperexcitability needs to be determined in further study.

$\mathrm{MeCbl}$ is regarded as a potential vitamin of neuropathic pain killer with excellent tolerance and safety at the moment [35]. Continuous treatment with high dose of MeCbl ameliorates mechanical allodynia via the inhibition of SA of 
A-type DRG neurons, the excitability, and $I_{h}$ current density of medium-sized DRG neurons in the animal model of low back pain. Thus, high dose MeCbl has great potential for treating peripheral neuropathy. Further investigations with $\mathrm{MeCbl}$ may help elucidate its mechanisms of action, which may further enable us to treat peripheral neuropathic pain.

\section{Conflict of Interests}

The authors declare that there is no conflict of interests regarding the publication of this paper.

\section{Authors' Contribution}

Ming Zhang, Wenjuan Han, and Jianyong Zheng contributed equally to this work.

\section{Acknowledgments}

The authors wish to thank Fei Da for his help with some of the purification of drugs. This work was supported by the National Natural Science Foundation of China (30870830 and 31371120 to Dr. Hui $\mathrm{Xu}$ ), the Ministry of Education Foundation for Returned Overseas Students (HG3503 to Dr. Hui Xu), National Natural Science Foundation (81272089), and the Natural Science Foundation of Shaanxi Province (21014JQ4127 to Mrs. Wenjuan Han).

\section{References}

[1] G. Scalabrino and M. Peracchi, "New insights into the pathophysiology of cobalamin deficiency," Trends in Molecular Medicine, vol. 12, no. 6, pp. 247-254, 2006.

[2] J. Metz, "Cobalamin deficiency and the pathogenesis of nervous system disease," Annual Review of Nutrition, vol. 12, pp. 59-79, 1992.

[3] N. V. Antoshkina, L. I. Vorob'eva, and E. P. Iordan, "Participation of methylcobalamin in methylation of DNA from Propionibacterium shermanii," Mikrobiologiya, vol. 48, no. 2, pp. 217-221, 1979.

[4] R. I. Kliasheva, E. V. Sharkova, I. I. Nikol'skaia, E. V. Belozerova, and A. M. Iurkevich, "Participation of methylcobalamin in DNA methylation in vitro," Biulleten' eksperimental'no ı̆ biologii i meditsiny, vol. 119, no. 6, pp. 616-618, 1995.

[5] A. Pfohl-Leszkowicz, G. Keith, and G. Dirheimer, "Effect of cobalamin derivatives on in vitro enzymatic DNA methylation: methylcobalamin can act as a methyl donor," Biochemistry, vol. 30, no. 32, pp. 8045-8051, 1991.

[6] K. Okada, H. Tanaka, K. Temporin et al., "Methylcobalamin increases Erk1/2 and Akt activities through the methylation cycle and promotes nerve regeneration in a rat sciatic nerve injury model," Experimental Neurology, vol. 222, no. 2, pp. 191203, 2010.

[7] Y. Nishikawa, S. Shibata, T. Shimazoe, and S. Watanabe, "Methylcobalamin induces a long-lasting enhancement of the field potential in rat suprachiasmatic nucleus slices," Neuroscience Letters, vol. 220, no. 3, pp. 199-202, 1996.

[8] A. McCaddon and P. R. Hudson, "L-methylfolate, methylcobalamin, and $\mathrm{N}$-acetylcysteine in the treatment of Alzheimer's disease-related cognitive decline," CNS Spectrums, vol. 15, no. 1, supplement 1, pp. 2-6, 2010.

[9] Y. Takahashi, S. Usui, and Y. Honda, "Effect of vitamin B12 (mecobalamin) on the circadian rhythm of rat behavior," Clinical Neuropharmacology, vol. 15, supplement 1, pp. 46A47A, 1992.

[10] S. L. B. Aashish and S. Morani, "Neuroprotective effect of early treatment with pioglitazone and methylcobalamin in alloxan induced diabetes in rats," Pharmacologyonline, vol. 3, pp. 282293, 2007.

[11] A. S. Morani and S. L. Bodhankar, "Early co-administration of vitamin $\mathrm{E}$ acetate and methylcobalamin improves thermal hyperalgesia and motor nerve conduction velocity following sciatic nerve crush injury in rats," Pharmacological Reports, vol. 62, no. 2, pp. 405-409, 2010.

[12] H. Ide, S. Fujiya, Y. Asanuma, M. Tsuji, H. Sakai, and Y. Agishi, "Clinical usefulness of intrathecal injection of methylcobalamin in patients with diabetic neuropathy," Clinical Therapeutics, vol. 9, no. 2, pp. 183-192, 1987.

[13] G. Li, "Effect of mecobalamin on diabetic neuropathies. Beijing Methycobal Clinical Trial Collaborative Group," Zhonghua Nei Ke Za Zhi, vol. 38, no. 1, pp. 14-17, 1999.

[14] P. M. Singh, M. Dehran, V. K. Mohan, A. Trikha, and M. Kaur, "Analgesic efficacy and safety of medical therapy alone vs combined medical therapy and extraoral glossopharyngeal nerve block in glossopharyngeal neuralgia," Pain Medicine, vol. 14, no. 1, pp. 93-102, 2013.

[15] J. Teramoto, "Effects of Methylcobalamin on neuralgia," Neurological Therapeutics, vol. 1, no. 2, p. 315, 1984.

[16] G. Xu, Z.-W. Lv, Y. Feng, W.-Z. Tang, and G. X. Xu, "A singlecenter randomized controlled trial of local methylcobalamin injection for subacute herpetic neuralgia," Pain Medicine, vol. 14, no. 6, pp. 884-894, 2013.

[17] K. Shibuya, S. Misawa, S. Nasu et al., "Safety and efficacy of intravenous ultra-high dose methylcobalamin treatment for peripheral neuropathy: a phase I/II open label clinical trial," Internal Medicine, vol. 53, no. 17, pp. 1927-1931, 2014.

[18] D. Vasudevan, M. M. Naik, and Q. I. Mukaddam, "Efficacy and safety of methylcobalamin, alpha lipoic acid and pregabalin combination versus pregabalin monotherapy in improving pain and nerve conduction velocity in type 2 diabetes associated impaired peripheral neuropathic condition. [MAINTAIN]: Results of a pilot study," Annals of Indian Academy of Neurology, vol. 17, no. 1, pp. 19-24, 2014.

[19] W. Waikakul and S. Waikakul, "Methylcobalamin as an adjuvant medication in conservative treatment of lumbar spinal stenosis," Journal of the Medical Association of Thailand, vol. 83, no. 8, pp. 825-831, 2000.

[20] C. A. Briggs and S. Chandraraj, "Variations in the lumbosacral ligament and associated changes in the lumbosacral region resulting in compression of the fifth dorsal root ganglion and spinal nerve," Clinical Anatomy, vol. 8, no. 5, pp. 339-346, 1995.

[21] S.-J. Hu and J.-L. Xing, "An experimental model for chronic compression of dorsal root ganglion produced by intervertebral foramen stenosis in the rat," Pain, vol. 77, no. 1, pp. 15-23, 1998.

[22] J.-M. Zhang, X.-J. Song, and R. H. Lamotte, "Enchanced excitability of sensory neurons in rats with cutaneous hyperalgesia produced by chronic compression of the dorsal root 
ganglion," Journal of Neurophysiology, vol. 82, no. 6, pp. 33593366, 1999.

[23] Z. Y. Tan, D. F. Donnelly, and R. H. LaMotte, "Effects of a chronic compression of the dorsal root ganglion on voltagegated $\mathrm{Na}^{+}$and $\mathrm{K}^{+}$currents in cutaneous afferent neurons," Journal of Neurophysiology, vol. 95, no. 2, pp. 1115-1123, 2006.

[24] H. Yao, D. F. Donnelly, C. Ma, and R. H. LaMotte, "Upregulation of the hyperpolarization-activated cation current after chronic compression of the dorsal root ganglion," Journal of Neuroscience, vol. 23, no. 6, pp. 2069-2074, 2003.

[25] C. Ma and R. H. LaMotte, "Enhanced excitability of dissociated primary sensory neurons after chronic compression of the dorsal root ganglion in the rat," Pain, vol. 113, no. 1-2, pp. 106112, 2005.

[26] J. Yagi and R. Sumino, "Inhibition of a hyperpolarizationactivated current by clonidine in rat dorsal root ganglion neurons," Journal of Neurophysiology, vol. 80, no. 3, pp. 10941104, 1998.

[27] R. S. Scroggs, S. M. Todorovic, E. G. Anderson, and A. P. Fox, "Variation in IH, IIR, and ILEAK between acutely isolated adult rat dorsal root ganglion neurons of different size," Journal of Neurophysiology, vol. 71, no. 1, pp. 271-279, 1994.

[28] C. G. Cardenas, L. P. del Mar, A. V. Vysokanov et al., "Serotonergic modulation of hyperpolarization-activated current in acutely isolated rat dorsal root ganglion neurons," Journal of Physiology, vol. 518, no. 2, pp. 507-523, 1999.

[29] S. R. Chaplan, H.-Q. Guo, D. H. Lee et al., "Neuronal hyperpolarization-activated pacemaker channels drive neuropathic pain," Journal of Neuroscience, vol. 23, no. 4, pp. 11691178, 2003.

[30] Y. Q. Jiang, G. G. Xing, S. L. Wang et al., "Axonal accumulation of hyperpolarization-activated cyclic nucleotide-gated cation channels contributes to mechanical allodynia after peripheral nerve injury in rat," Pain, vol. 137, no. 3, pp. 495-506, 2008.

[31] Q. Sun, G.-G. Xing, H.-Y. Tu, J.-S. Han, and Y. Wan, "Inhibition of hyperpolarization-activated current by ZD7288 suppresses ectopic discharges of injured dorsal root ganglion neurons in a rat model of neuropathic pain," Brain Research, vol. 1032, no. 1-2, pp. 63-69, 2005.

[32] M. D. Boada and C. J. Woodbury, "Physiological properties of mouse skin sensory neurons recorded intracellularly in vivo: Temperature effects on somal membrane properties," Journal of Neurophysiology, vol. 98, no. 2, pp. 668-680, 2007.

[33] S. R. Chaplan, F. W. Bach, J. W. Pogrel, J. M. Chung, and T. L. Yaksh, "Quantitative assessment of tactile allodynia in the rat paw," Journal of Neuroscience Methods, vol. 53, no. 1, pp. 55-63, 1994.

[34] S.-J. Hu, H.-J. Yang, Z. Jian et al., "Adrenergic sensitivity of neurons with non-periodic firing activity in rat injured dorsal root ganglion," Neuroscience, vol. 101, no. 3, pp. 689-698, 2000.

[35] M. Zhang, W. Han, S. Hu, and H. Xu, "Methylcobalamin: a potential vitamin of pain killer," Neural Plasticity, vol. 2013, Article ID 424651, 6 pages, 2013.

[36] S. Yagihashi, A. Tokui, H. Kashiwamura, S. Takagi, and K. Imamura, "In vivo effect of methylcobalamin on the peripheral nerve structure in streptozotocin diabetic rats," Hormone and Metabolic Research, vol. 14, no. 1, pp. 10-13, 1982.

[37] T. Watanabe, R. Kaji, N. Oka, W. Bara, and J. Kimura, "Ultrahigh dose methylcobalamin promotes nerve regeneration in experimental acrylamide neuropathy," Journal of the Neurological Sciences, vol. 122, no. 2, pp. 140-143, 1994.

[38] Z.-B. Wang, Q. Gan, R. L. Rupert, Y.-M. Zeng, and X.-J. Song, "Thiamine, pyridoxine, cyanocobalamin and their combination inhibit thermal, but not mechanical hyperalgesia in rats with primary sensory neuron injury," Pain, vol. 114, no. 1-2, pp. 266277, 2005.

[39] L. Jian-bo, W. Cheng-Ya, C. Jia-Wei, L. Xiao-Lu, F. Zhen-Qing, and M. Hong-Tai, "The preventive efficacy of methylcobalamin on rat peripheral neuropathy influenced by diabetes via neural IGF-1 levels," Nutritional Neuroscience, vol. 13, no. 2, pp. 79-86, 2010.

[40] H. Mizukami, S. Ogasawara, S.-I. Yamagishi, K. Takahashi, and S. Yagihashi, "Methylcobalamin effects on diabetic neuropathy and nerve protein kinase C in rats," European Journal of Clinical Investigation, vol. 41, no. 4, pp. 442-450, 2011.

[41] Q.-P. Ma and C. J. Woolf, "Progressive tactile hypersensitivity: an inflammation-induced incremental increase in the excitability of the spinal cord," Pain, vol. 67, no. 1, pp. 97-106, 1996.

[42] S. Neumann, T. P. Doubell, T. Leslie, and C. J. Woolf, "Inflammatory pain hypersensitivity mediated by phenotypic switch in myelinated primary sensory neurons," Nature, vol. 384, no. 6607, pp. 360-364, 1996.

[43] K. Noguchi, Y. Kawai, T. Fukuoka, E. Senba, and K. Miki, "Substance $\mathrm{P}$ induced by peripheral nerve injury in primary afferent sensory neurons and its effect on dorsal column nucleus neurons," The Journal of Neuroscience, vol. 15, no. 11, pp. 76337643, 1995.

[44] N. Fan, D. F. Donnelly, and R. H. la Motte, "Chronic compression of mouse dorsal root ganglion alters voltage-gated sodium and potassium currents in medium-sized dorsal root ganglion neurons," Journal of Neurophysiology, vol. 106, no. 6, pp. 30673072, 2011.

[45] P. J. Waddell, S. N. Lawson, and P. W. McCarthy, "Conduction velocity changes along the processes of rat primary sensory neurons," Neuroscience, vol. 30, no. 3, pp. 577-584, 1989.

[46] G. Maccaferri and C. J. McBain, "The hyperpolarizationactivated current (Ih) and its contribution to pacemaker activity in rat CA1 hippocampal stratum oriens-alveus interneurones," Journal of Physiology, vol. 497, no. 1, pp. 119-130, 1996.

[47] A. A. Nava-Ocampo, A. Pastrak, T. Cruz, and G. Koren, "Pharmacokinetics of high doses of cyanocobalamin administered by intravenous injection for 26 weeks in rats," Clinical and Experimental Pharmacology and Physiology, vol. 32, no. 1-2, pp. 13-18, 2005.

[48] E. E. Benarroch, "HCN channels: function and clinical implications," Neurology, vol. 80, no. 3, pp. 304-310, 2013.

[49] H. Tu, L. Deng, Q. Sun, L. Yao, J.-S. Han, and Y. Wan, "Hyperpolarization-activated, cyclic nucleotide-gated cation channels: roles in the differential electrophysiological properties of rat primary afferent neurons," Journal of Neuroscience Research, vol. 76, no. 5, pp. 713-722, 2004.

[50] L. L. Gao, S. McMullan, L. Djouhri, C. Acosta, A. A. Harper, and S. N. Lawson, "Expression and properties of hyperpolarizationactivated current in rat dorsal root ganglion neurons with known sensory function," Journal of Physiology, vol. 590, no. 19, pp. 4691-4705, 2012.

[51] C. Acosta, S. McMullan, L. Djouhri et al., "HCN1 and HCN2 in Rat DRG neurons: levels in nociceptors and non-nociceptors, 
NT3-dependence and influence of CFA-induced skin inflammation on HCN2 and NT3 expression," PLoS ONE, vol. 7, no. 12, Article ID e50442, 2012.

[52] S. Schnorr, M. Eberhardt, K. Kistner et al., "HCN2 channels account for mechanical (but not heat) hyperalgesia during longstanding inflammation," Pain, vol. 155, no. 6, pp. 1079-1090, 2014. 

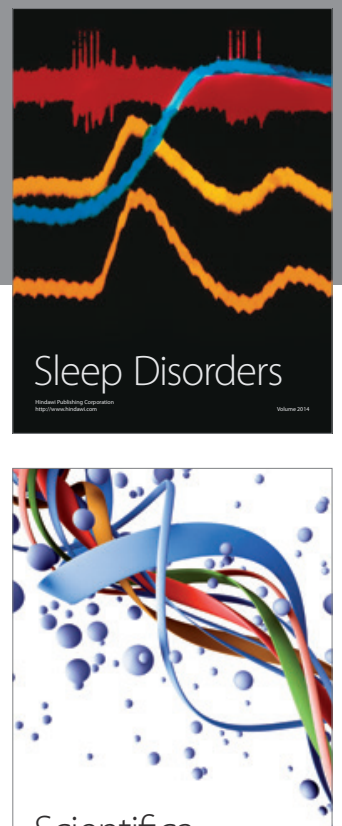

Scientifica
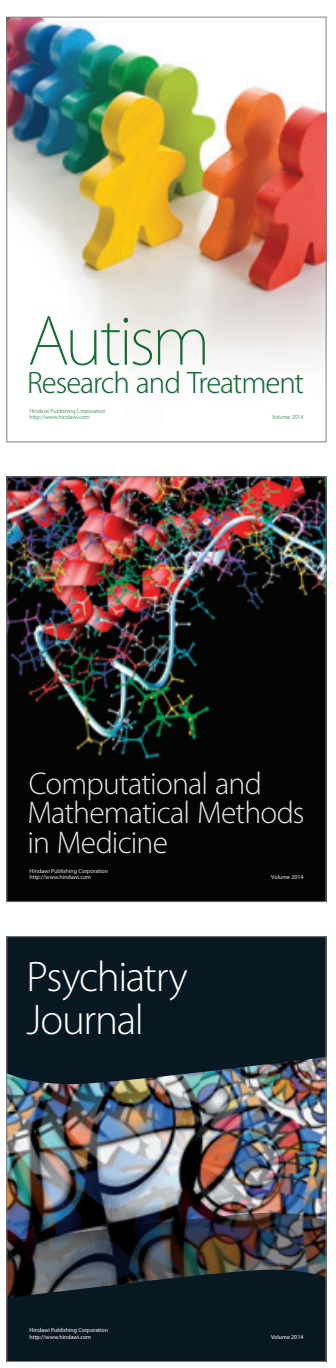
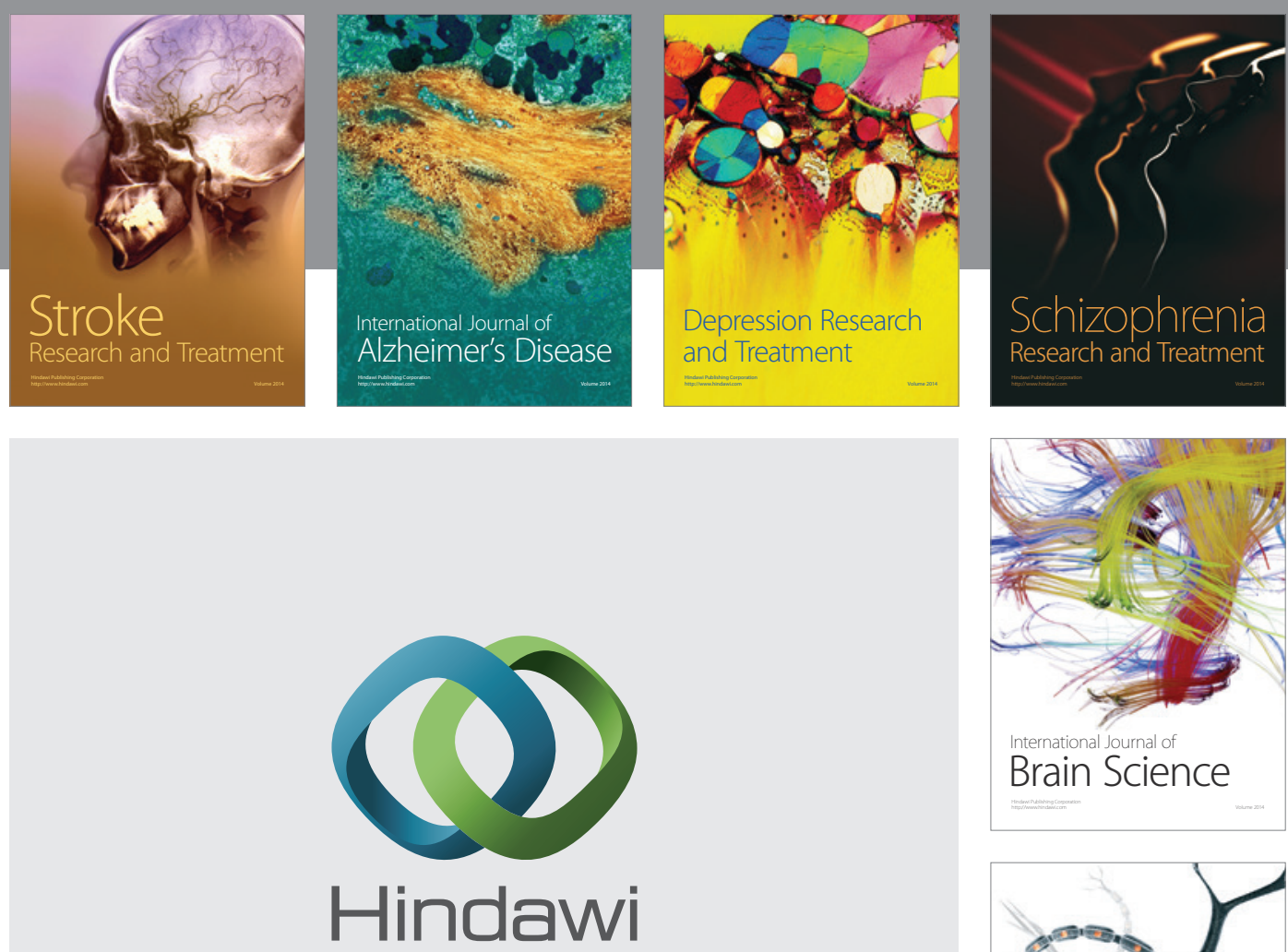

Submit your manuscripts at

http://www.hindawi.com
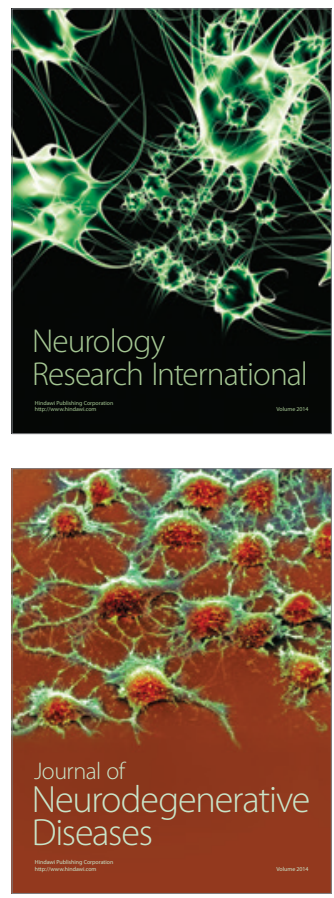

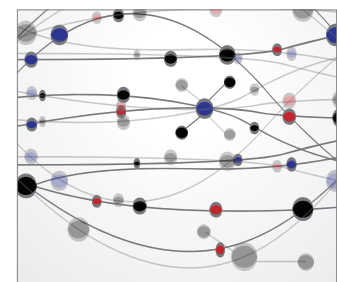

The Scientific World Journal
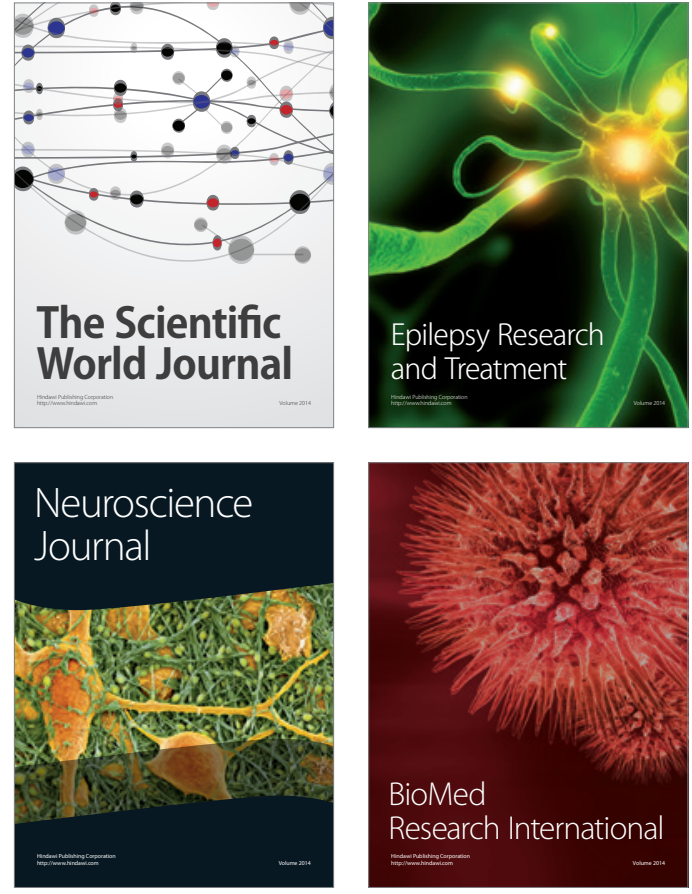

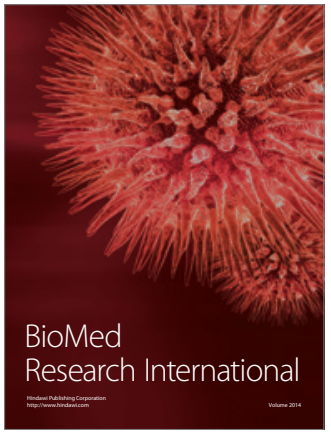

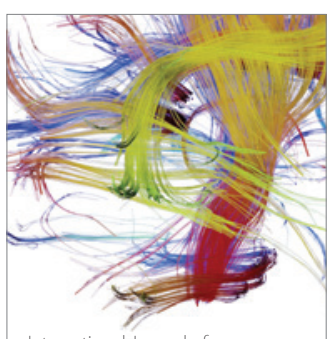

Brain Science

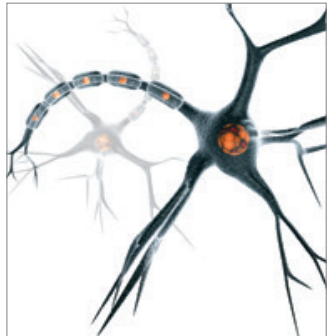

Neural Plasticity
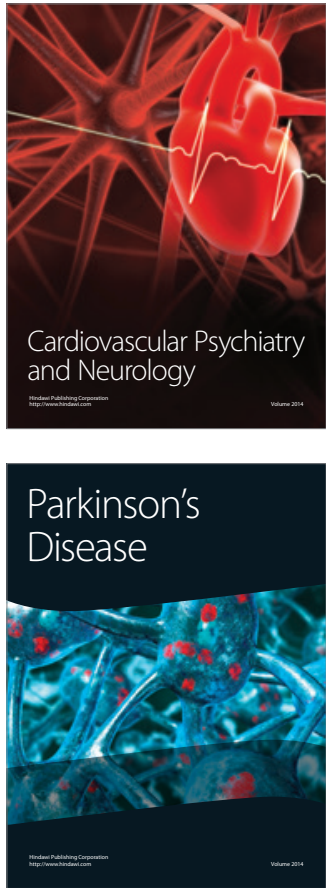